\title{
An Approach to 1/f Noise Detection Based on Adaptive T-ATFPF Algorithm
}

\author{
Jie Wu ${ }^{1,2}$, Xiaojuan Chen ${ }^{1 *}$, Zhaohua Zhang ${ }^{1}$ \\ ${ }^{I}$ College of electronic information engineering, Changchun University of Science and Technology, \\ Changchun 130022, China \\ ${ }^{2}$ Beihua University, School of electrical and information engineering, Jilin 132021, China \\ "Corresponding Author.
}

\begin{abstract}
The generation of $1 / f$ noise is closely related to the quality defects of IGBT devices. In the process of detecting IGBT single tube noise, thermal noise and shot noise show obvious white noise characteristics in the low frequency band, which are detected under the background of strong white noise $1 / f$ noise can characterize the performance of IGBT devices. Therefore, on the basis of the Time-Frequency Peak Filtering (TFPF) algorithm, a two-dimensional time-domain adaptive T-ATFPF algorithm is proposed, and the adaptive segmentation is realized by means of the confidence interval crossing criterion based on Chebyshev's inequality. Variable window length, use a small window length to process the signal section, which retains more detailed information of the effective signal.Use a larger window length to process the buffer section to ensure a smooth transition. Use the large window length to process the noise section, which more effectively suppresses randomness for noise, apply T-ATFPF to artificial synthesis model and actual model. Experimental results indicate that compared with the conventional algorithm, the improved method can better recover $1 / f$ noise, and the ratio of signal to noise is greatly improved by about $1.3 d B$
\end{abstract}

Keywords:1/fnoise,time-frequencypeakfiltering, two-dimensional time-domain adaptive filtering

\section{I.Introduction}

As science and technology develop, electronic equipment has been widely utilized in many fields. In high-tech fields such as nuclear power generation, aerospace and military industry, and submarine exploration, the reliability of electronic equipment is extremely demanding. As an important component of electronic equipment, semiconductor devices are closely related to system stability. However, semiconductor devices are in batches Devices with potential defects are inevitably produced in production. These devices have a short service life, and have major hidden dangers to the stability of the system. Once a failure occurs, it may cause irreparable losses. Therefore, the reliability analysis methods of semiconductor devices have gained extensive attention from many scholars domestically and abroad. In recent years, the research methods of reliability testing have mainly focused on the aging test method and the noise analysis method. The noise analysis method has the characteristics of short test time in comparison with the aging test method, low equipment requirements, high test efficiency and no damage to the test device, and has become the mainstream analysis and research method. $1 / f$ noise is the main part of low-frequency noise in semiconductor devices, and its characteristics are closely linked to the internal defects of the device. As a consequence, in-depth exploration of $1 / f$ noise characteristics is of remarkable significance for device reliability analysis.

IGBT (Insulated Gate Bipolar Transistor) is a power semiconductor device composed of BJT and MOS. It is currently the most technologically advanced product in power electronic devices. It is widely used in high-precision fields such as high-speed rail, aviation, electric vehicles, and wind power generation. Traditional IGBT operation reliability research is to observe the changes of related parameters in the aging or failure process of IGBT devices, and then evaluate the health status of the devices or predict the remaining life[1-2]. M. Fukuda et al.[3-4] found through research that for semiconductor devices with PN junctions, the low-frequency noise is mainly $1 / f$ noise. The generation of $1 / f$ noise closely linked to the defects and impurities of the device, and its power spectral density is obvious and easy to observe in the low-frequency brackets. Therefore, the reliability of the device can be indirectly

ISSN: 0010-8189

(C) CONVERTER 2021 
reflected by studying the low-frequency noise[5-6]. In the process of detecting IGBT single-tube noise, thermal noise and shot noise exhibit obvious white noise characteristics in the low-frequency scope. Therefore, how to accurately detect $1 / f$ noise under the background of strong white noise is the research topic of this article.

Time-Frequency Peak Filter (TFPF) is a noise elimination algorithm widely used in the field of signal processing $[7-8]$. And it has been proved that the noise cancellation can still be achieved effectively under the condition of low SNR[9]. Based on the signal enhancement method of instantaneous frequency estimation, a time-frequency peak filtering algorithm is proposed. The noise-containing signal is encoded into the instantaneous frequency of the frequency-modulated analytical signal through frequency modulation, and its time-frequency distribution is concentrated along the instantaneous frequency. The crest value of the time-frequency distribution of the frequency-modulated analytical signal is estimated as the instantaneous frequency, thereby eliminating random noise[10].

In recent years, researchers around the world have continuously explored the problem of reducing strong random noise and improving the SNR, and have achieved practical results. Such as controllable direction filter[11], sparse denoising[12], particle filter[13], variational modal decomposition[14], Shearlet[15] and deep convolutional network[16] and other methods. With the windowed Wigner-Ville distribution (WVD), namely PWVD[17]. In this case, the time-frequency peak filtering technique can process the non-stationary signal at very low SNR. This paper adopts the time-frequency peak filter algorithm to realize the effective detection of $1 / f$ noise.

Firstly, the traditional TFPF algorithm is proved to be equivalent to a low pass filter through theoretical derivation, and its explicit filtering expression is obtained. We found that the traditional TFPF processing of random signals only uses a single window length, while the large window length is suitable for suppressing random noise, and the small window length is suitable for restoring effective signals, which cannot compromise consideration. So here we have the time domain adaptive TFPF, namely T-ATFPF. T-ATFPF uses the confidence interval crossover criterion based on Chebyshev's inequality to achieve adaptive segmental variable window length, use a small window length to process the signal segment, and more retain the detailed information of the effective signal, use a larger window length to process the buffer Sections ensure a smooth transition; use large window lengths to process noise sections, which can suppress random noise more effectively and obtain a higher SNR.

\section{II.Methodology}

\section{$2.11 / f$ noise theory}

Noise is a very common phenomenon in nature, it is the fluctuation of physical quantity over time. The internal noise of a semiconductor is macroscopically manifested as the phenomenon of voltage fluctuations at both ends of the semiconductor device. Its generation mechanism is directly have a bearing on the internal structure of the device. The noise generated by devices with different structures has different distinguishing feature, but the classification of these noises is generally the same. The quality of semiconductors is closely related to their low-frequency noise characteristics. A large number of experiments have shown that the low-frequency noise of semiconductor components give rise to surface damage or missing internal crystal lattices. Semiconductor internal noise can be delimited white noise and low-frequency noise. The former is mainly composed of thermal noise and shot noise, and the latter mainly includes G-R noise and $1 / f$ noise.

The characteristic of $1 / f$ noise is that its power spectral density changes negatively exponentially with increasing frequency. At present, most scholars believe that $1 / f$ noise theory and models are generally based on two fluctuation mechanisms, the surface carrier number fluctuation mechanism and the carrier number migration mechanism[18]. The $1 / f$ noise power spectral density can be expressed as

ISSN: 0010-8189

(C) CONVERTER 2021 


$$
S_{x}(w) \sim \frac{\sigma_{x}^{2}}{|\omega|^{\gamma}}
$$

Where $S_{x}(w)$ is signal power spectral density, $\sigma_{x}^{2}$ is the variance of the signal, $\gamma$ is the frequency factor.

From the above analysis, it can be known that the thermal noise power spectral density and shot noise is not affected by frequency, and cannot be used as an index to measure the quality and reliability of semiconductor devices. In these noise analysis methods, we should try to restrain or even filter the influence of the two on the experimental results. Impact. The mechanism of G-R noise and 1/f noise have a bearing on to the impurity and defect of the device, especially the characteristics of $1 / f$ noise power spectral density in the low-frequency scopes are intuitive and easy to distinguish, therefore, low-frequency noise often reflects the intrinsic quality and reliability of the device, and is the best choice for measuring the quality and reliability of semiconductor devices. The research on $1 / f$ noise in the existing noise analysis method is particularly important.

$1 / f$ noise mainly has two important characteristics, statistical self-similarity and long range dependence. Among them, the statistical selfsimilarity is a time-domain feature, which means that the $1 / f$ noise time-domain waveform is approximately the same under different observation scales. Assuming that the input signal is $x(t), a$ is the scale factor, and $H^{\prime}$ is the similarity index, then:

$$
x(t)^{P}=a^{-H^{\prime}} x(a t)
$$

According to formula (2), the $1 / f$ noise power spectral density expression (3) can be obtained as follows:

$$
S_{x}(\omega)=|\partial|^{\gamma} S_{x}(a \omega)
$$

In the formula, $\gamma=2 H^{\prime}+1$, the $1 / f$ noise power spectral density at different scales only differs by a fixed amplitude factor $|\partial|^{\gamma}$. Long-range correlation is the characteristic of long-term persistence. From the generalized inverse Fourier transform of Equation (3), the autocorrelation function of $1 / f$ noise can be obtained as follows:

$$
R x(\tau)=\frac{\tau^{\gamma-1}}{2 \Gamma(\gamma) \cos (\gamma \pi / 2)} \sim|\tau|^{\gamma-1}
$$

In the formula, $\Gamma$ is a function of Gamma, $\tau$ is a time delay. From the analysis of formula (4), $R_{x}(\tau)$ decreases slowly with $\tau$, the 1/f noise has a long-range correlation in the time domain.

\subsection{Time domain adaptation (T-ATFPF)}

\subsubsection{TFPF}

TFPF is a nonlinear signal smoothing algorithm based on instantaneous frequency (IF) estimation. Taking advantage of the good energy accumulation of the windowed WVD, the instantaneous frequency of the noise-containing signal after frequency modulation is estimated for denoising.

In this paper, the detection of $1 / f$ noise in the IGBT studied can be modeled by the following formula:

ISSN: 0010-8189 


$$
s=x+g
$$

Among them, $x \in R^{n \times 1}$ is the effective signal without noise, which in this article refers to the $1 / f$ noise we need to recover. $g \in R^{n \times 1}$ is random noise, in this article, refers to the Gaussian white noise we added.And $s \in R^{n \times 1}$ is the noisy observation signal. First, the noisy signal $s$ is frequency-modulated to make it the IF of the frequency-modulated signal:

$$
z_{i}=\exp \left(i 2 \pi \mu \sum_{j=0}^{i} s_{j}\right)
$$

Among them, $\mu>0$ is the frequency modulation coefficient. Then, calculate $P W V D$ about $z$ :

$$
(W)_{i, f}=\sum_{\tau=-L}^{L} \omega_{\tau} z_{i-\tau}^{*} z_{i+\tau} \exp (-i 4 \pi f \tau)
$$

Among them, $W \in R^{n}$ is the window function, and its length is $W \in R^{n}$. Finally, the instantaneous frequency at the peak is obtained from $(W)_{i, f}$ as an estimate of $x$ :

$$
\hat{x}=\arg \max _{f}\{W\} / \mu
$$

According to the above three steps, TFPF can finally be equivalent to a low-pass filter, expressed as the following form:

$$
\begin{gathered}
\hat{x}=h * s \\
h_{i}=-\sum_{\tau=-L}^{i} \tau \omega_{\tau} / \sum_{\tau=-L}^{L} \tau^{2} \omega_{\tau}
\end{gathered}
$$

Among them, * represents the convolution operation, $h \in R^{n}$ represents the impulse response of $T F P F$, and has the same length as the window function $\omega$.

\subsubsection{T-ATFPF}

Chebyshev's inequality was proposed by the famous Russian mathematician Chebyshev in the 19th century. The specific definition is as follows: If the random variable $\mathrm{X}$ has finite variance, then for any positive number ${ }^{\varepsilon}$ :

$$
P\{\mid X-E[X] \geq \varepsilon\} \leq \frac{D[X]}{\varepsilon^{2}}
$$

Among them, $E[X]$ is the mean of $X$, and $D[X]$ is the variance of $X$. It shows that when the variance $D[X]$ is smaller, the random variable $X$ is denser around the mathematical expectation $E[X]$. Therefore, variance can be used to describe the degree of deviation of random variables from their mathematical expectations [19], and Chebyshev's inequality can be used to estimate the probability of random variables falling into a certain 
interval.

The realization of the principle of confidence interval crossing is as follows: First, introduce a rectangular window sequence $K=\left\{k_{1}<k_{2} \cdots<k_{J}\right\}$ with $\mathrm{J}$ increasing window lengths, and perform TFPF on each sampling point; then, bring the estimated value obtained into equation (12):

$$
P\left\{\left|\hat{x}_{k}(n)-x(n)\right|<\varepsilon\right\} \geq 1-\frac{D\left[e_{k}(n)\right]}{\varepsilon^{2}}
$$

Solve to obtain the confidence interval corresponding to the window length; finally, use the confidence interval intersection to obtain the filter window length $k_{i} \in K$ at this point, $k_{i}$ is the maximum window length that satisfies $D k_{i}(n) \cap D k_{i+1}(n)=\varnothing$ in the rectangular window sequence. In other words, $k_{i}$ is the maximum value in $K$ that satisfies the confidence interval $D_{k_{i}}(n)$ and $D_{k_{i+1}}(n)$ has no common point[20]. The confidence interval intersection schematic diagram is shown in Fig.1.

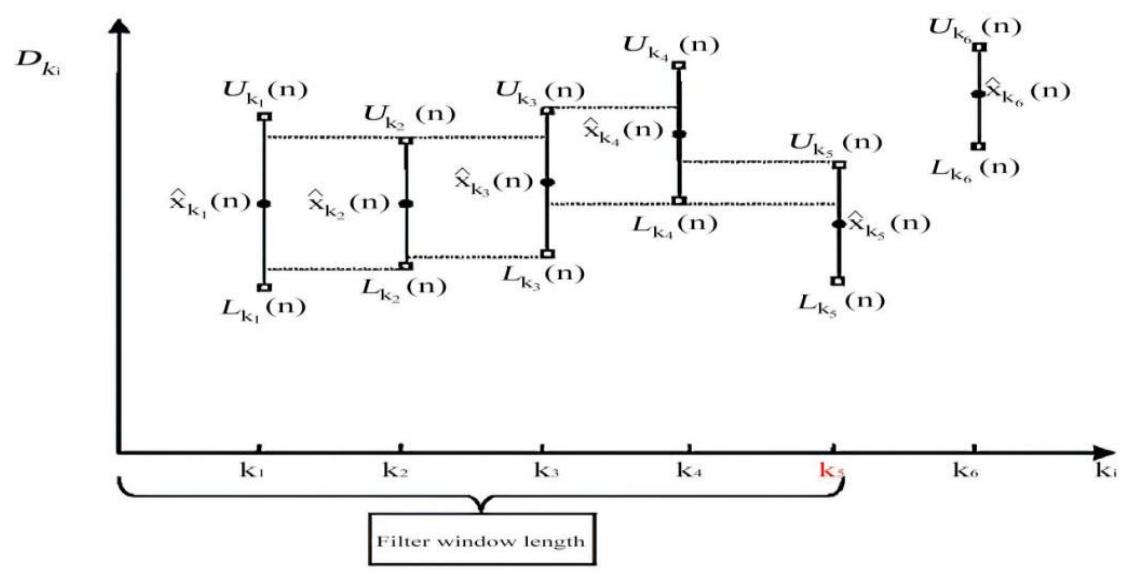

Fig. 1:Confidence interval intersection schematic diagram

Based on the above principles, this paper proposes a time-domain adaptive TFPF (T-ATFPF). The specific implementation steps are as follows:

First, the filter window length of each point obtained by using the confidence interval crossing criterion is used as an intermediate variable, and the short-term energy is calculated by combining the following formula:

$$
E_{n}=\sum_{m=1}^{N}[h(m) \omega(n-m)]^{2}
$$

Among them, $E_{n}$ is the short-term energy of the intermediate variable of the sampling point $n, \omega(m)$ is the rectangular window function, $h(m)$ is the intermediate variable corresponding to the sampling point $m$, and $\mathrm{N}$ is the number of sampling points. The basic frequency is $25 \mathrm{~Hz}$, the sampling frequency is $500 \mathrm{~Hz}$, and the rake wavelet with $-9 \mathrm{~dB}$ Gaussian white noise is added as input. The filter window length and its normalized short-term energy are

ISSN: 0010-8189 
obtained by using the confidence interval crossover criterion as shown in Fig. 2.

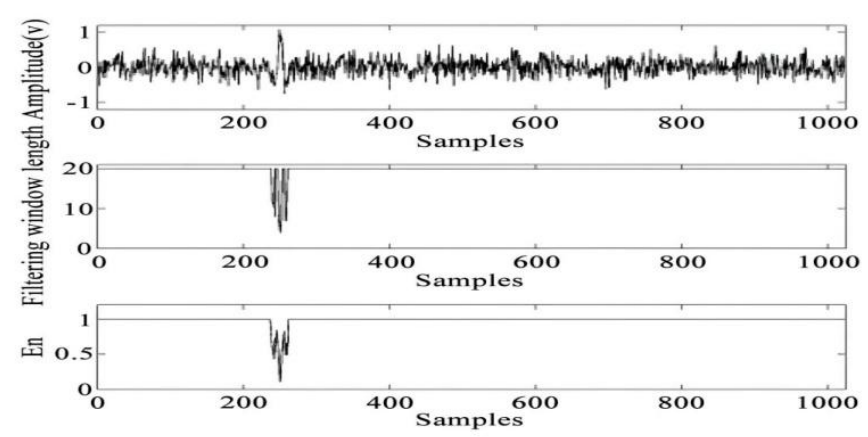

Fig. 2: Noisy signal, filter window length and normalized short-term energy

Then, from the short-term energy value distribution chart obtained above, select a threshold value $\lambda$ to segment the noisy signal. The specific segmentation principle is as follows: when $E_{n}<\lambda, s(n)$ belongs to the signal segment; when $E_{n} \geq \lambda$, if $s(n)$ is at both ends of the signal segment and the range is less than half of the signal segment length, then $s(n)$ belongs to the buffer segment. Otherwise, $s(n)$ belongs to the noise segment. According to the actual situation of the signal, $\lambda$ can be selected to be processed. The adaptive segmentation diagram of noisy signal is shown in Fig. 3.

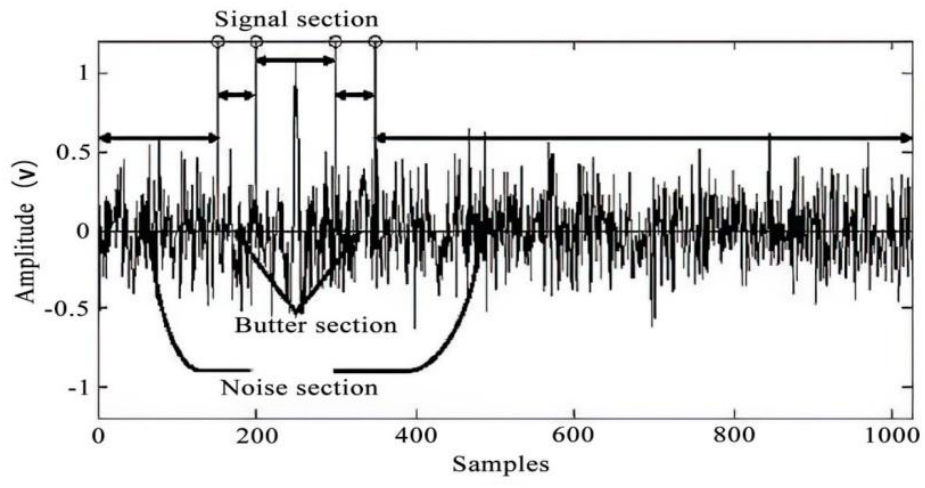

Fig. 3: Schematic diagram of adaptive segmentation

Next, use the empirical window length formula to calculate the optimal filter window length of the signal segment. The length formula of empirical window is as follows:

$$
W L=\frac{0.353 f_{s}}{f_{d}}
$$

Among them, $f_{s}$ is the sampling frequency, and $f_{d}$ is the dominant frequency of the signal segment ${ }^{[21]}$. It can be obtained by performing Fourier transform on the signal segment, and $f_{d}$ is the frequency spectrum at the peak. The buffer section selects a larger window length for smooth transition, such as $W L+2$ sampling points, and the noise section directly selects a large window length for noise suppression.

Finally, superimpose the filtering results of the signal section, buffer section and noise section to obtain the final

ISSN: 0010-8189

(C) CONVERTER 2021 
filtering result.

\section{III.Simulation Results and Analysis}

\subsection{Artificially synthesized $1 / f$ noise model}

First, a synthetic $1 / f$ noise model is given to test the denoising ability of TFPF. The fractional Brownian motion model has fractal characteristics, long-range correlation and power-law spectrum characteristics, and can simulate the $1 / f$ noise model well [21-22], as shown in formula (15).

$$
B_{H}(t)-B_{H}(0)=\frac{1}{\Gamma(H+1 / 2)} \int_{-\infty}^{t} K\left(t-t^{\prime}\right) d B\left(t^{\prime}\right)
$$

The kernel function:

$$
K\left(t-t^{\prime}\right)=\left\{\begin{array}{c}
\left(t-t^{\prime}\right)^{H-1 / 2}, 0 \leq t \leq t \\
\left(t-t^{\prime}\right)^{H-1 / 2}-(-t)^{H-1 / 2}, h^{\prime}<0
\end{array}\right.
$$

In the formula, the parameters $0<H<1$ and $B(t)$ are ordinary Gaussian stochastic processes with a mean value of 0 , and set $\left\langle B^{2}(t)\right\rangle=t$.

This article mainly uses matlab software platform, uses fractional Brownian model to simulate, the noise obtained by setting $\mathrm{H}=0.35$ is shown in Fig. 4.

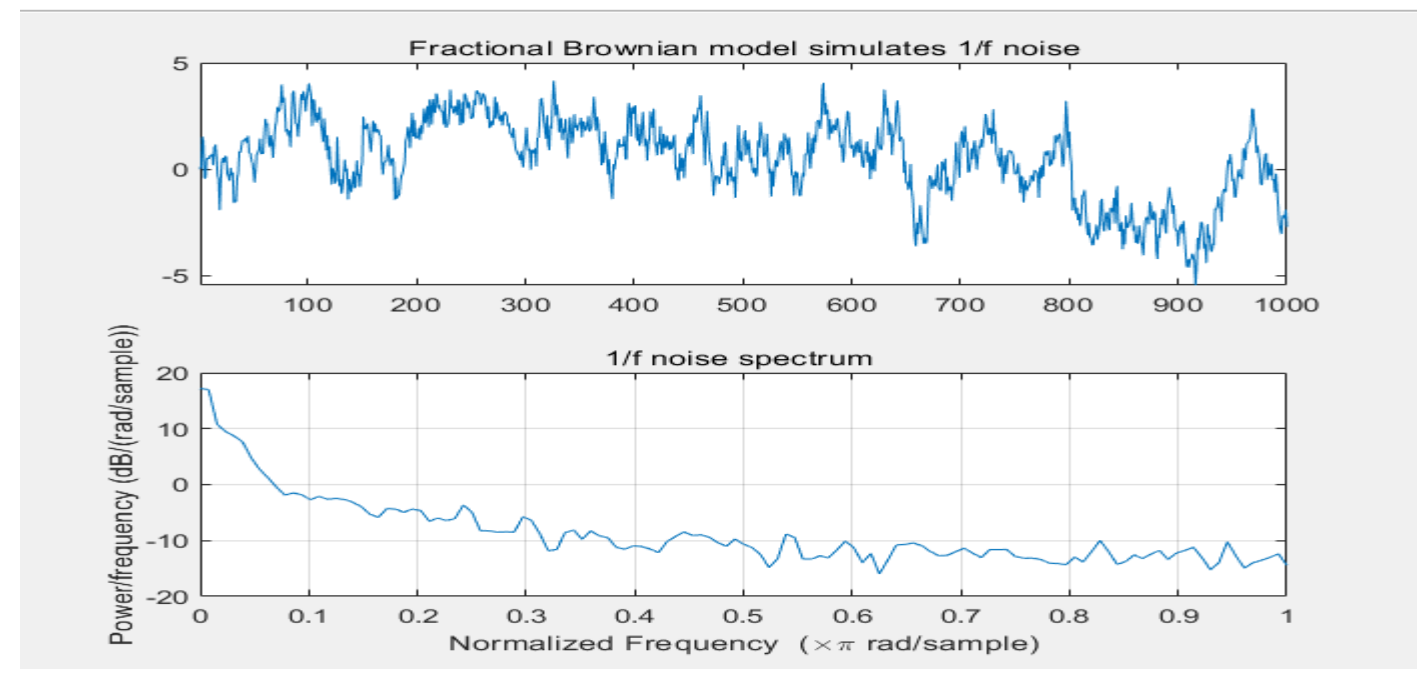

Fig. 4: Fractional Brownian model simulation of 1/f noise

For better simulate the real situation, add white noise with a power spectral density of $1.44 \mathrm{~dB}$ to the $1 / \mathrm{f}$ noise, as shown in Fig. 5. In the light of Fig. 5, the original 1/f noise signal is enhanced, which helps to better observe the noise detection effect of this algorithm. 


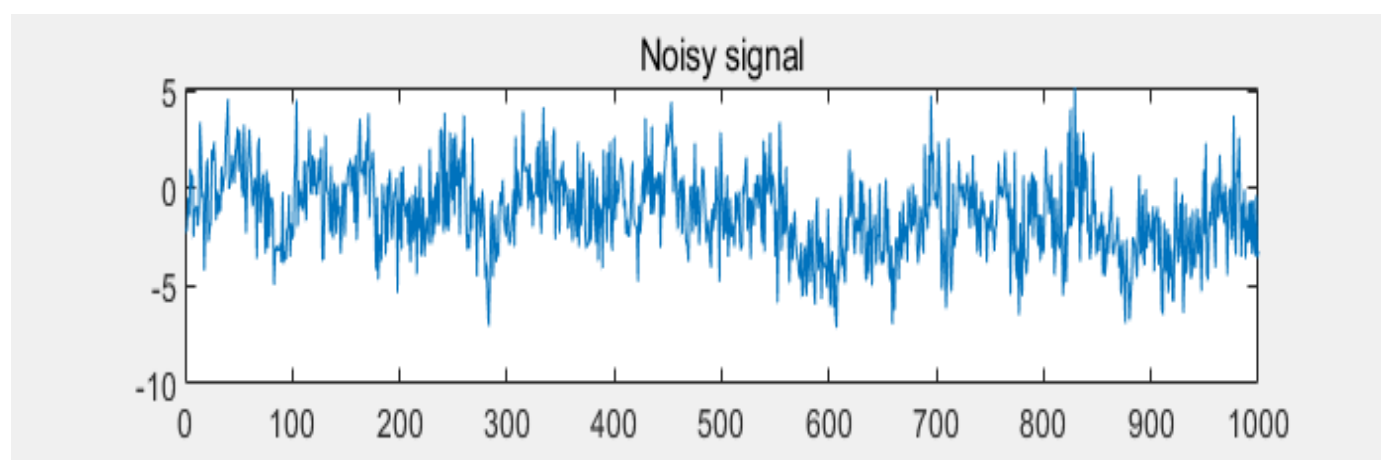

Fig. 5: 1/f noise after adding noise

The traditional TFPF and the two-dimensional adaptive T-ATFPF are used to process the noisy signal respectively. The window length of the traditional TFPF is set to 9 points. The TFPF filter set parameters used in the latter method ensure a reasonable comparison: $H=\left\{h_{1}, h_{1499}\right\}$, corresponding the rectangular window is 1 - and 1499-point data length respectively, and the passband of this group of filters is sufficient to cover the entire signal spectrum. In order to make the comparison effect more obvious, we use the traditional TFPF with a window length 9 , the two-dimensional adaptive control T-ATFPF to process them respectively. When the directional derivative parameter $\mathrm{n}$ of the two-dimensional T-ATFPF is from 1 to 100, the output the change of the SNR is shown in Fig. 6. It can be seen that $\mathrm{n}$ achieves the maximum output SNR at 10. The filtering results are shown in Fig. 7 and Fig. 8, respectively. Comparing the results of two algorithms, it can be find out that the denoising effect of TFPF under the background of strong actual noise is not obvious, and there is still much random noise remaining, while the two-dimensional adaptive T-ATFPF can better restore the original noise signal. The noise effect is more obvious and the background is cleaner, which more powerfully shows that T-ATFPF is better than traditional TFPF in effective signal recovery, frequency tracking and noise suppression.

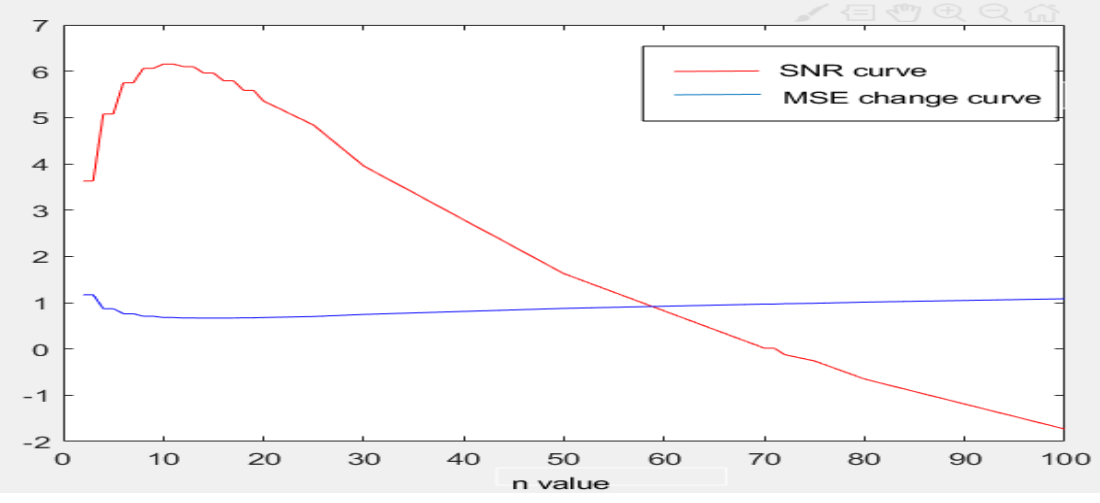

Fig. 6: Output signal-to-noise ratio and mean square error curve 


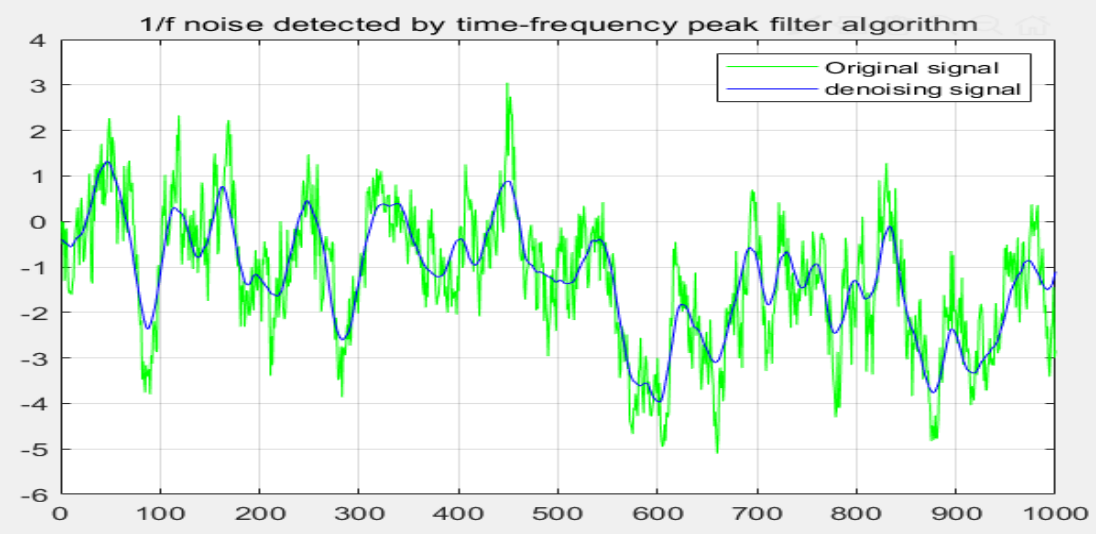

Fig. 7: Traditional TFPF restores 1/f noise

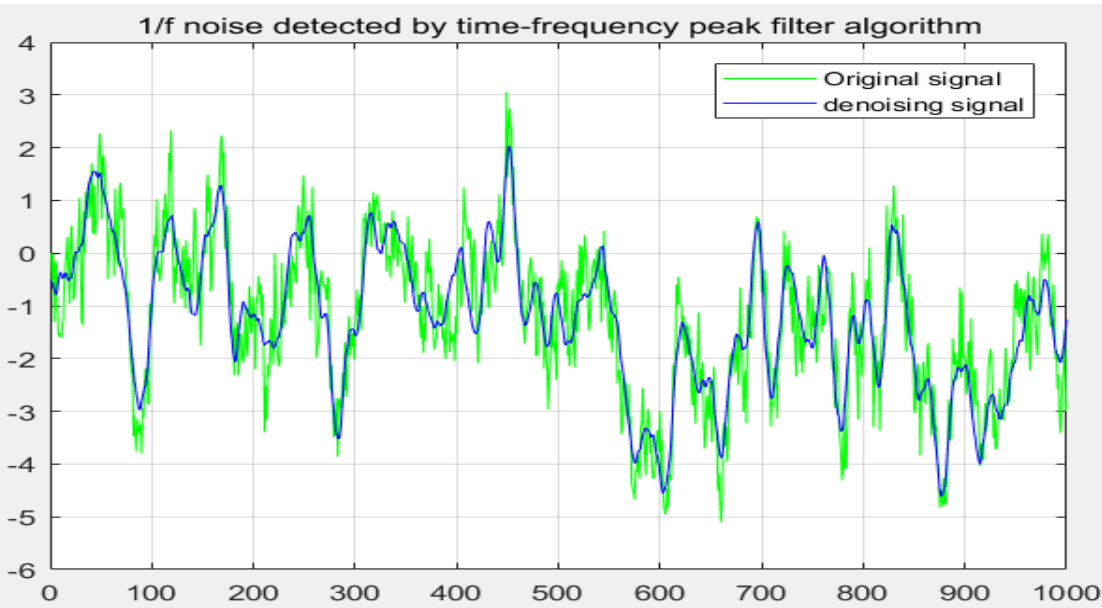

Fig. 8: Two-dimensional adaptive T-ATFPF recovering $1 /$ f noise

In order to more accurately measure the detection effects between different algorithms, according signal-to-noise ratio (SNR) and mean square error (MSE) to selected the evaluation parameters, as shown in formulas (17) and (18). Comparing the different signal-to-noise ratio (SNR) and mean square error (MSE) of the two methods, the results are showing in Table 1. The larger the SNR parameter, the better the denoising effect of the algorithm, and the smaller the MSE, the more similar the detected signal is to the original signal. On the basis of the fractional Brownian model simulation of $1 / f$ noise, Gaussian white noise of $0 \mathrm{~dB},-3 \mathrm{~dB}$ and $-5 \mathrm{~dB}$ are added respectively. This table further illustrates the superiority of T-ATFPF in noise suppression.

$$
\begin{array}{r}
S N R=10 \times \log _{10}\left(\sum_{i}^{N} \frac{y_{i}^{2}}{\left(x_{i}-y_{i}\right)^{2}}\right) \\
M S E=\frac{1}{N} \sum_{i=1}^{N}\left(x_{i}-y_{i}\right)^{2}
\end{array}
$$

Table 1 SNR comparison before and after filtering

\begin{tabular}{|c|c|c|c|c|}
\hline $\begin{array}{c}\text { SNR before } \\
\text { filtering }(\mathrm{dB})\end{array}$ & $\begin{array}{c}\text { SNR after } \\
\text { TFPF }(\mathrm{dB})\end{array}$ & MSE after TFPF & $\begin{array}{c}\text { SNR after } \\
\text { T-ATFPF(dB) }\end{array}$ & $\begin{array}{c}\text { MSE after } \\
\text { T-ATFPF }\end{array}$ \\
\hline 0 & 1.6258 & 0.8760 & 3.3851 & 1.0786 \\
\hline-3 & 2.8355 & 0.7004 & 4.2954 & 0.9106 \\
\hline-5 & 3.6287 & 0.5789 & 5.6045 & 0.7369 \\
\hline
\end{tabular}




\subsection{1/f noise in actual IGBT devices}

In the previous section, through the simulation experiment of the simulated results of $1 / f$ noise model, it can be seen that the T-ATFPF is very effective for the analog signal. This section verifies the applicability of the two-dimensional adaptive T-ATFPF method to reduce random noise by processing the $1 / f$ noise in the actual IGBT device. In an environment with a temperature dimension of 25 degrees Celsius,set the starting frequency to $1 \mathrm{~Hz}$, the cut-off frequency to $102400 \mathrm{~Hz}$, and the sampling frequency to $100 \mathrm{~Hz}$. The TFPF with a window length 9 and the two-dimensional adaptive T-ATFPF are used to process them, and the simulation results are as follows as shown in Fig. 9 and Fig. 10.

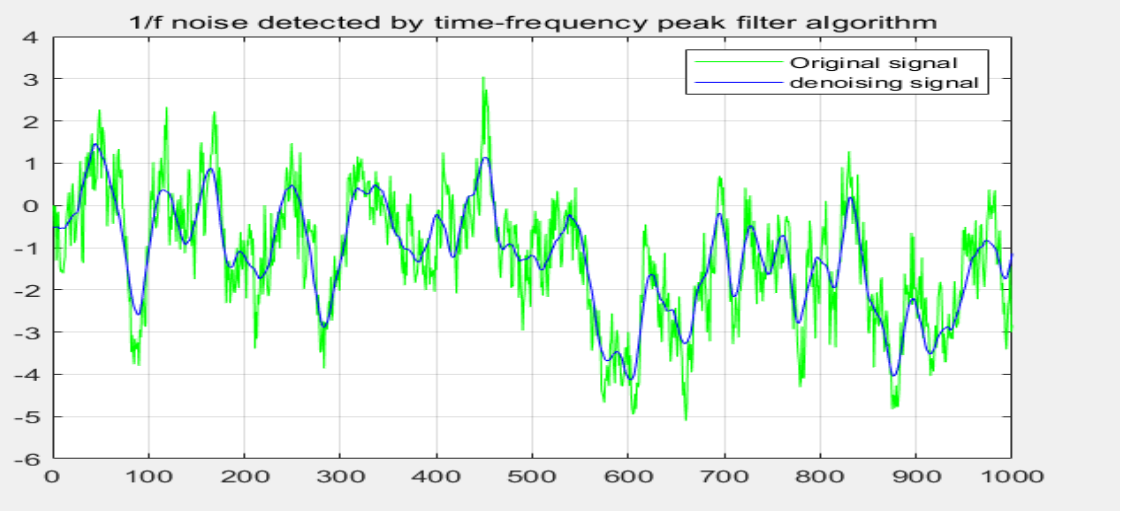

Fig. 9: Traditional TFPF detects 1/f noise

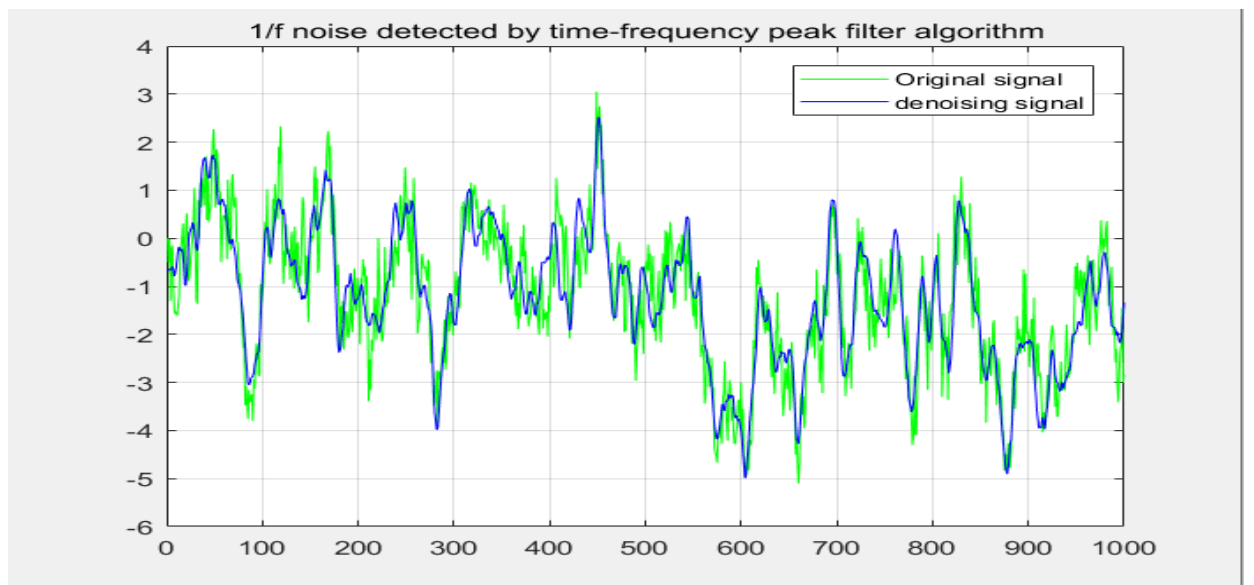

Fig. 10: Two-dimensional adaptive T-ATFPF detects 1/f noise

Comparing the $1 / f$ noise signal models recovered by the two, it is not difficult to find that the denoising effect after the two-dimensional adaptive T-ATFPF processing is clearer and better.

\section{IV.Conclusion}

As the most advanced power semiconductor device at present, IGBT devices are widely used in high-tech and high-cost fields such as aerospace and rail transportation. Their quality is related to huge economic losses and even casualties. Therefore, how to accurately detect the quality and reliability of IGBT devices is very important. The noise analysis method is widely used because of its fast, effective, lossless and other characteristics. Based on the traditional TFPF algorithm in this paper, a two-dimensional adaptive T-ATFPF is proposed using the confidence interval crossing criterion based on Chebyshev's inequality. First obtain the confidence interval of each point; then use the crossover principle to obtain the filter window length of each point; Second use the obtained filter window

ISSN: 0010-8189 
length as an intermediate variable to obtain its short-term energy, and divide the noisy signal into signal section, buffer section and Noise section; Finally, the empirical window length formula is used to obtain the optimal filter window length of the signal section to complete the adaptive segmented filtering. Through simulated recording and actual recording simulation experiments, it can be seen that the improved two-dimensional adaptive T-ATFPF has a better effect on random noise suppression and effective signal recovery.

\section{Acknowledgements}

This work was supported in part by the National Natural Science Foundation of China (Serialnumberoftheproject:61271115), and in part byEducationDepartmentofJilinProvince (Serialnumberoftheproject: 201696).

\section{References}

[1] M.Musallam,C.M.Johnson,C.Y.Yin,et al.,"In-service life consumption estimation in power modules,"Proceedings of the 13th International Power Electronics and Motion Control Conference, New York,USA,2008

[2] S.Yang, D.Xiang,A.Bryant, et al.,"Condition monitoring for device reliability in power electronicconverters:A review," IEEE Transactions on Power Electronics, vol. 25, no.11, pp. 2734-2752, 2011.

[3] M. Fukuda, T. Hirono, T. Kurosakiand, et al.,"Correlation between $1 / f$ noise and a semiconductor laser degradation,” Qual Reliab Engng Inter, vol. 10, pp.351-353, 1994.

[4] M. Fukuda, T. Hirono, T. Kurosakiand, et al.,"1/f noise behavior in semiconductor laser degradation," IEEE Photon, Tech. Lett, vol. 5, pp. 1165-1171, 1993.

[5] K.Alicja, M.W.Bogdan, “Noise in Semiconductor Devices,” Fundamentals of Industrial Electronics, no. 11, pp.1-12, 2010.

[6] W.Hei,"Low-frequency noise study in electron devices:review and update," Microelectronics Reliability,vol. 43,pp.585-599, 2003.

[7] H. Lin, Y. Li, B. Yang, H. Ma, C. Zhang, "Seismic random noise elimination by adaptive time-frequency peak filtering," IEEE Geoscience and Remote Sensing Letters (S1545-598X), vol. 11, no. 1, pp. 337-341, 2014.

[8] Y. Tian, Y. Li,"Parabolic-trace time-frequency peak filtering for seismic random noise attenuation," IEEE Geoscience and Remote Sensing Letters (S1545-598X), vol. 11, no. 1, pp. 158-162, 2014.

[9] B. Boashash, M. Mesbah, "Signal enhancement by time-frequency peak filtering," IEEE Transactions on Signal Processing (S1053-587X), vol. 52, no. 4, pp. 929-937, 2004.

[10] H.B. Lin, Y. Li, B.J. Yang, H.T. Ma,“Seismic Random Noise Elimination byAdaptive Time-Frequency Peak Filtering, IEEE Geoscience and Remote Sensing Letters (S1545-598X), vol. 11, no. 1, pp. 337-341, 2014.

[11] P-L. Shui, H-Y. Shang, Y-B. Zhao,"Instantaneous frequency estimation based on directionallysmoothed pseudo-Wigner-Ville distribution bank," IET Radar Sonar Navig (S1751-8784), vol. 1, no. 4, pp. 317-325, 2007.

[12] M.H.Huang, Y.Li, "EHGFs direction controllable filtering suppresses random noise in seismic exploration,” Journal of Jilin University: Information Science Edition, vol. 34, no. 3, pp.310-314,2016.

[13] Y.B.Bu, N.S. Liang, D.Shao, et al.,"Desert seismic noise reduction based on compound sparse denoising. Journal of Jilin University: Information Science Edition, vol. 36, no. 3, pp. 240-245, 2018.

[14] Y.M.Liu, S.Y.Gao, "Application of hybrid particle swarm algorithm in seismic impedance inversion," Journal of Jilin University: Information Science Edition, vol. 36, no. 5, pp. 403-410, 2018.

[15] X.Liu,W. Li, “Correlation energy entropy adaptive threshold denoising based on variational modal decomposition," Journal of Jilin University: Information Science Edition, vol. 37, no. 5, pp. 518-525, 2019. 
[16] J.Q. Gong, N.Wu,"First arrival picking of microseismic based on Shearlet-AIC algorithm,"Journal of Jilin University: Information Science Edition, vol. 36, no. 3, pp. 233-239, 2018.

[17] S.Zheng, Y. Li, X.T.Dong,"Shearlet domain depth residual CNN is used for desert seismic signal denoising," Journal of Jilin University: Information Science Edition, vol. 31, no. 1, pp. 1-7, 52, 2018.

[18] B.BOASHASH,M.MESBAH,"Signal Enhancement by Time-Frequency Peak Filtering,"IEEE Transactions on Signal Processing,vol. 52, no. 4, pp. 929-937, 2004.

[19] L.K.J.Vandamme, “1/f noise in MOS devices, mobility or number fluctuations,"Electron Devices,IEEE Transactions on, vol. 41, no. 11, pp. 1936-1945, 1994.

[20] F.Principato, G.Ferrante,"Skewnessandkurtosisof1/fnoiseinsemiconductor devices,"Microelectronics Reliability, vol. 40, no. 11, pp. 1969-1973, 2000.

[21] G.H. Zhuang, Y. Li, Y.P.Liu, H. B. Lin, H. T. Ma, N. Wu,"VaryingWindow-LengthTFPFinHigh-ResolutionRadonDomainforSeismicRandom Noise Attenuation,"IEEE Geoscienceand Remote Sensing Letters, vol. 12, no. 2, pp. 404-408, 2015.

[22] X.H. Deng, H.T. Ma, Y. Li, Q. Zeng, "Seismic random noise attenuation based on adaptive time-frequency peak filtering," Journal of Applied Geophysics, vol. 113, pp. 31-37, 2015. 